\title{
Microengineering of Collagen Hydrogels Integrated into Microfluidic Devices for Perfusion Culture of Mammalian Cells
}

\author{
Misaki Kato, Mayu FuKushi, Masumi YamadA*, Rie UtOH, and Minoru SEKI \\ Department of Applied Chemistry and Biotechnology, Graduate School of Engineering, Chiba University, 1-33 Yayoi-cho, Inage-ku,
}

Chiba 263-8522, Japan

\begin{abstract}
Collagen-based hydrogels are widely used for three-dimensional (3D) culture of mammalian cells because of their high cell-activating characteristis. However, techniques for preparing of cell-embedding collagen hydrogels with micrometer-size precision in perfusable, microfluidic devices have not been fully developed. In this study, we propose a facile strategy enabling microfabrication of collagen hydrogels in microfluidic devices. We used phosphate particle-embedding polydimethylsiloxane (PP-PDMS) as a gelation agent, which neutralizes the acidic collagen soltuion. The collagen solution near the surface of the PP-PDMS is selectively gelled. We fabricated micropatterns and tubular structures made of collagen hydrogel, both of which were used for perfusion culture of mammalian cells encapsulated in the hydrogel matrix. The presented approach would be applicable to various types of cell culture experiments.
\end{abstract}

\section{Introduction}

In recent years, researchers have developed perfusion culture devices of mammalian cells, which can reproduce the blood circulation system in the organs or tissues in our body. In an attempt to provide tissue or organ models, socalled organs-on-a-chip devices or microphysiological systems have been intensively studied, to improve the efficiency of drug development. Many types of perfusion systems have utilized 2D culture systems for cell culture, some of which utilize permeable membranes for the cell adhesion substrate. (Walter et al., 2016; Salerno et al., 2018; Iwadate et al., 2019). These systems can mimic the molecular transport through the basement membranes and to reconstruct the heterotypic cell-cell interactions.

Despite the great promise of these perfusion culture systems, they do not realize complex cell-matrix interactions. In our body, cells are surrounded by extracellular matrix (ECM) components, including collagen, laminin, fibronectin, and so on. The use of these ECM-based hydrogels was proven to be significantly effective for culturing specific cell types, as represented by hepatocytes. Therefore, it was considered that the incorporation of the hydrogel components into perfusion culture systems would be highly useful to enhance and/or regulate the cell function, viability, differentiation, and proliferation. However, it was not easy to form hydrogel patterns or hydrogel tubes into microfluidic devices that can encapsulate living cells in the hydrogel matrices.

In this study, we propose a facile and versatile strategy to form microengineered hydrogel structures composed of type I collagen in polydimethylsiloxane (PDMS)-based microfluidic systems. The concept of formation of collagen hydrogels on the surface of PDMS substrate is shown in Figure 1 (Furusawa et al., 2012). We employed phosphate particle (PP)-embedding PDMS (PP-PDMS) as the material of the microfluidic device. When an acidic collagen solution was dropped onto or contacted with the surface of the PP-PDMS, the phosphate particles were dissolved, resulting in the partial neutralization of the collagen solution and the hydrogel formation at the vicinity of the PP-PDMS surface. In this study, this mechanism was applied to the formation of collagen micropatterns around the PP-PDMS posts in PDMS microchannels. Additionally, we created tubular collagen structures in channels entirely composed of PP-PDMS (entirely PP-PDMS channel). Both of these structures were used to perform perfusion culture of mammalian cells embedded in the collagen hydrogel matrix, to investigate the usefulness of our approach for cell culture applications.

\section{Experimental Section}

\subsection{Fabrication of PP-PDMS microchannels}

We fabricated two types of microfluidic devices partially made of PP-PDMS; (1) flat PDMS channels equipped with PP-PDMS microposts for hydrogel patterning, and (2) channels entirely composed of PP-PDMS for creating tubular collagen hydrogels. To produce the PP-PDMS micropost-incorporating channels, we first fabricated SU8 molds on a silicon wafer using a standard photolithography technique. Then, PDMS prepolymer

Corresponding author: m-yamada@faculty.chiba-u.jp 
with embedded $\mathrm{Na}_{2} \mathrm{HPO}_{4}$ particles (diameter of less than $25 \mu \mathrm{m})$ at a concentration of $30 \%(\mathrm{w} / \mathrm{w})$ was poured onto the holes of the mold, and it was heat-cured at $85^{\circ} \mathrm{C}$ for 10 min. Thereafter, pure PDMS prepolymer without containing the phosphate particles was poured onto the entire region of the mold, and heat-cured at $85^{\circ} \mathrm{C}$ for an additional $30 \mathrm{~min}$. The cured PDMS was peeled off from the mold and a PDMS plate having PP-PDMS microposts was obtained. The plate was bonded against a flat glass slide via $\mathrm{O}_{2}$ plasma treatment, to obtain the microchannel having PP-PDMS microposts.

To produce the entirely PP-PDMS channels, we used a PDMS mold with a convex structure for the microchannel. PP-PDMS prepolymer was poured onto the channel region of the mold, and it was cured at $85^{\circ} \mathrm{C}$ for $10 \mathrm{~min}$. Thereafter, pure PDMS prepolymer was poured onto the entire mold and cured for an additional $30 \mathrm{~min}$. The obtained PDMS plate was bonded against flat PPPDMS plate to prepare a microchannel, of which the inner surface was entirely made of PP-PDMS.

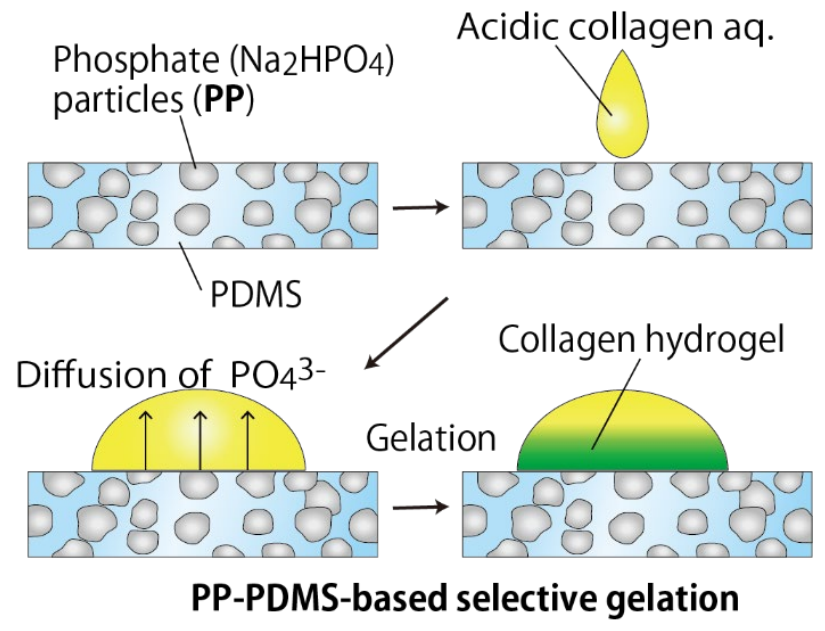

Figure 1. A schematic image showing the gelation process of an acidic collagen solution on the phosphate particleembedding PDMS (PP-PDMS) substrate

\subsection{Preparation of collagen hydrogels in microchannels}

Figure 2 shows the preparation process of the hydrogel patterns in flat channels with PP-PDMS microposts. First, $0.5 \%$ acidic type I collagen solution was introduced into the channel. When the channel was completely filled with the collagen solution, the flow was stopped and it was incubated for several minutes. An aqueous solution of $10 \%$ dextran as the washing buffer was then introduced into the channel to remove the non-gelled collagen solution. For creating collagen hydrogel tubes in the entirely PP-PDMS channels, the collagen solution was kept flowing for several minutes, and then the non-gelled solution was removed similarly. To observe the formation behaviors of the collagen hydrogel layer in the channels, green fluorescent microparticles $(\Phi$ of $1-3 \mu \mathrm{m})$ were introduced into the collagen solution in advance.
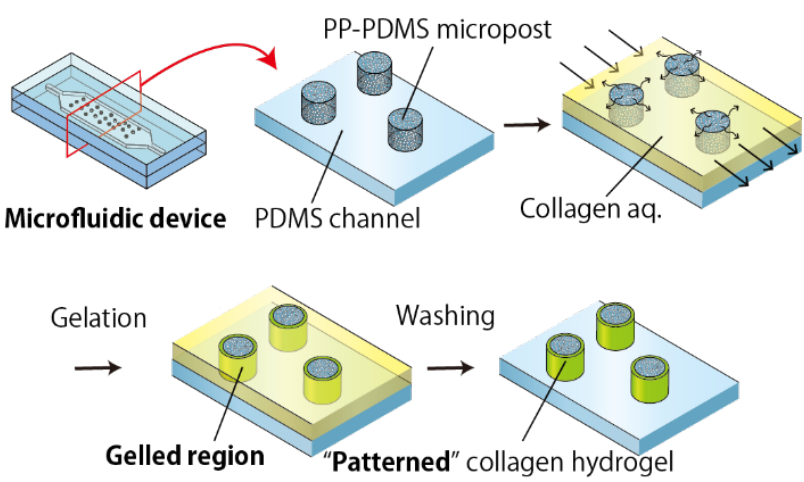

Figure 2. Fabrication process of the PP-PDMS micropostincorporating microfluidic channels and the collagen microgels around the posts of the PP-PDMS

\subsection{Cell culture experiments}

HepG2 cells (human hepatoma cell line) were suspended in the collagen solution, and then it was introduced into the microchannel with PP-PDMS microposts at a flow rate of $\sim 100 \mu \mathrm{L} / \mathrm{min}$. The non-gelled collagen solution was washed away with $10 \%$ dextran solution. After forming the hydrogel patterns with encapsulated cells, the cell culture medium was introduced using a syringe pump at a feed speed of $10 \mu \mathrm{L} / \mathrm{min}$. Perfusion culture was performed for up to 7 days at $37^{\circ} \mathrm{C}$ in a humidified $\mathrm{CO}_{2}$ incubator.

In addition, the entirely PP-PDMS channels were used for creating tubular collagen hydrogels, containing smooth muscle cells (SMCs), to produce blood vessel-like tissue models. SMCs were suspended in a collagen solution by the same procedure as described above, and the cell-containing collagen hydrogel tube was fabricated in the flow channel. Endothelial cells (ECs) were then introduced into the lumen. Perfusion culture was performed for up to 5 days in a humidified $\mathrm{CO}_{2}$ incubator.

\section{Results and Discussion}

\subsection{Observation of gelation on the PP-PDMS plate}

First, we investigated if the collagen hydrogels were actually formed on the surface of the PP-PDMS because of the interaction with the embedded phosphate particles. Figure 3 shows the collagen hydrogel formed on the PPPDMS plate. We confirmed that the gelation of the collagen solution took place on the PP-PDMS surface because the acidic collagen solution was neutralized by the embedded PP. We also confirmed that the thickness of the collagen hydrogel could be changed by adjusting the phosphate concentration and/or the incubation time period; the higher concentration of PP and the longer incubation time resulted in the formation of a thicker hydrogel layer. At high PP concentration conditions, the amount of PP present on the PDMS surface was increased, which was attributed to the increased hydrogel thickness. SEM observation revealed that the density of the microholes, which were formed by the dissolution of PP, 
increased after the hydrogel formation with the increase in the PP concentration. In the following experiments we fixed the PP concentration in the PDMS prepolymer at $30 \%(\mathrm{w} / \mathrm{w})$.

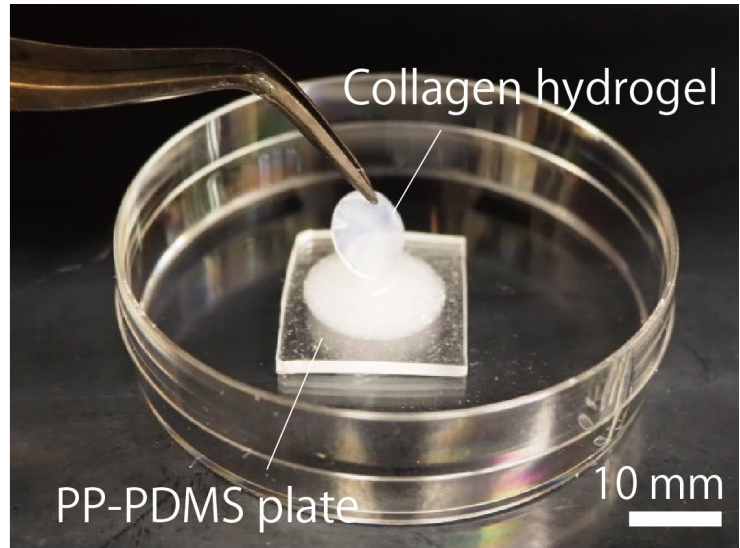

Figure 3. Photograph of the collagen hydrogel formed on a flat PP-PDMS plate.

\subsection{Preparation of PP-PDMS microchannels}

Next we prepared microchannel structures partially composed of PP-PDMS. Figure 4 shows several types of microchannels. We successfully prepared microchannels having PP-PDMS microposts with various shapes, and channels whose lumen was entirely composed of the PPPDMS matrix. For the PP-PDMS microposts, phosphate particles with a size less than $25 \mu \mathrm{m}$ were used so that these particles could be easily and uniformly incorporated into the microposts.
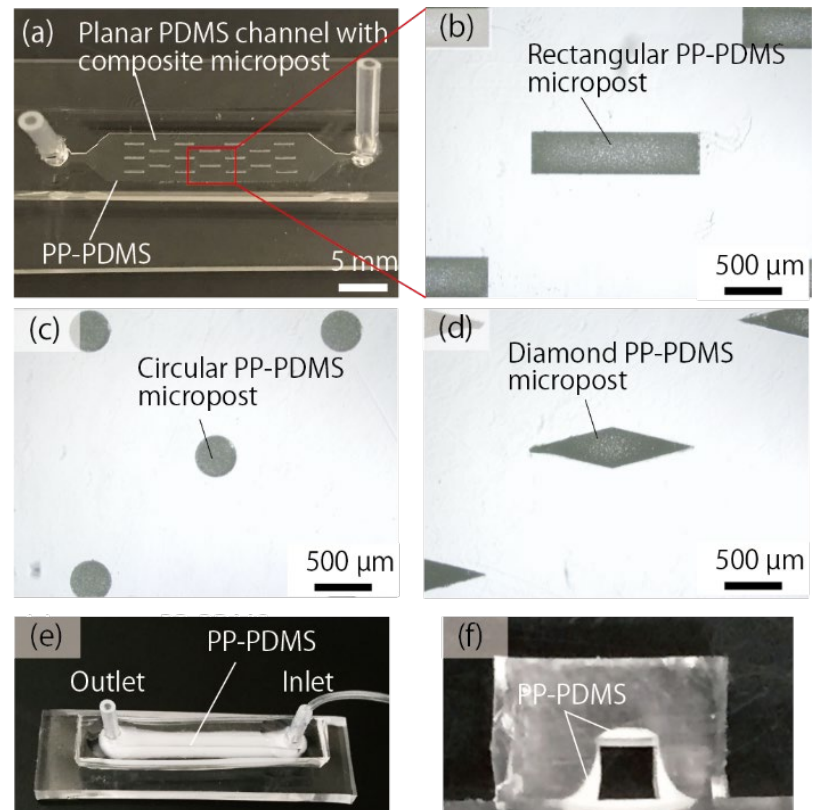

Linear channel structure

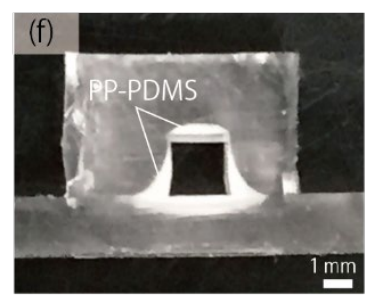

Figure 4. (a-d) Microchannels with PP-PDMS microposts with various shapes; (e, f) Microchannel entirely composed of PP-PDMS for creating tubular collagen hydrogels

\subsection{Formation of collagen hydrogels in microchannels}

We examined if micrometer-sized collagen structures were actually formed in the microchannels. Figure 5 shows the formation process of the collagen hydrogel around the PP-PDMS microposts. The hydrogels were started to form after $\sim 1$ min of introduction, when the flow of the collagen solution was stopped. The acidic collagen solution, introduced into the channel, was transformed into the hydrogel, selectively near the PP-PDMS region. After 2 min, a hydrogel layer with a thickness of $\sim 500 \mu \mathrm{m}$ was formed.

Next the entirely PP-PDMS channels were tested to form collagen hydrogel tubes on the lumen of the channels composed of PP-PDMS (Figure 6). After introducing a washing buffer, tubular collagen hydrogels were formed. The thickness of the hydrogel layer could be controlled by changing the input flow rate of the collagen solution and the gelation period (Figure $6(\mathrm{c})$ ). The higher flow rate resulted in the formation of the thinner hydrogel tube, because of the high shear force under the high flowrate condition.
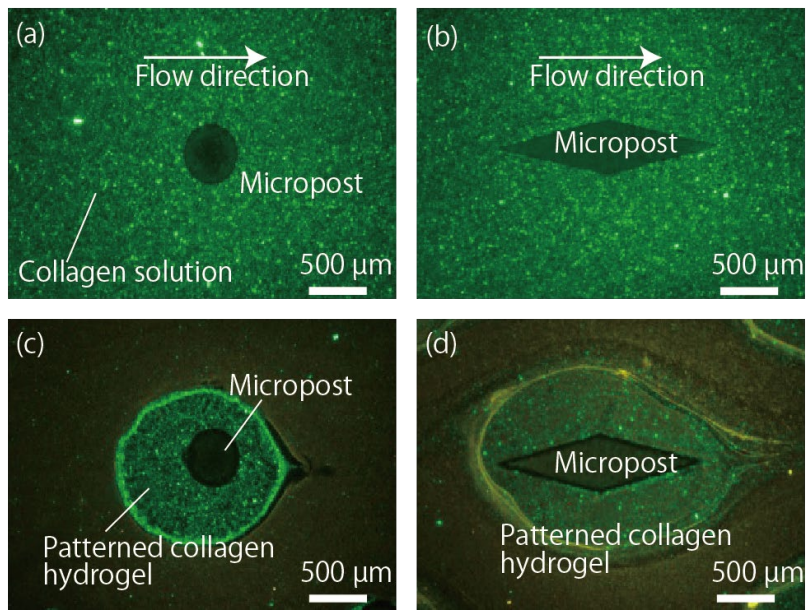

Figure 5. (a, b) Flow of the collagen solution with green fluorescent microparticles through the microchannel with the PP-PDMS microposts; (c, d) Formed collagen hydrogel structures around the circular and diamond microposts 

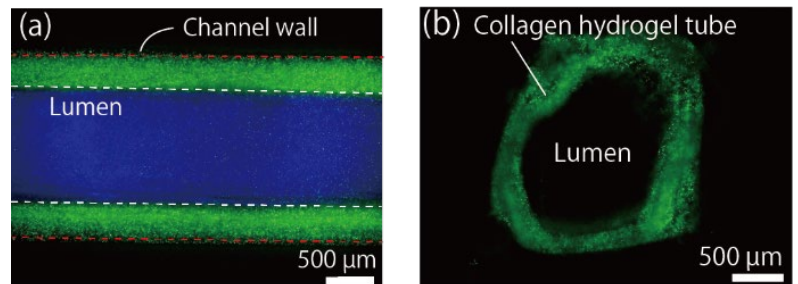

(c)

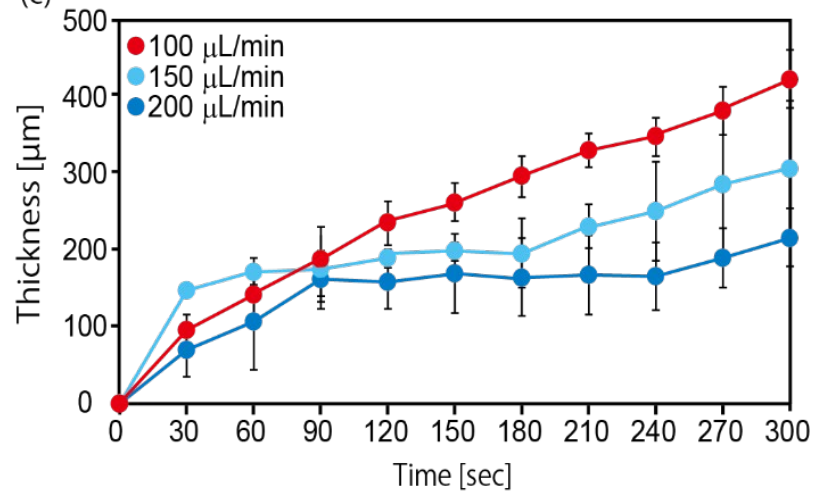

Figure 6. (a) Fluorescent micrograph showing the formed hydrogel layer (green) and the flow of the washing buffer (blue) in the entirely PP-PDMS microchannel; (b) Cross section of the formed hydrogel microtube; (c) Relation between the hydrogel thickness and the incubation time period, when the flow rate was changes as indicated

\subsection{Cell encapsulation and cultivation in the hydrogel}

Finally we attempted to encapsulate living mammalian cells in the hydrogel matrix to perform perfusion culture of the cells. Cells were suspended in the $0.5 \%$ acidic typeI collagen solution and introduced into the microchannel, to prepare cell-containing collagen hydrogel structures. In the channels with the PP-PDMS microposts, HepG2 cells were encapsulated and perfusion culture was performed for 7 days. During the perfusion culture, we confirmed that most of the cells were viable and proliferative (Figure 7 (a-d)).

For the tubular hydrogels, SMCs were introduced into the hydrogel matrix, whereas the ECs were seeded on the lumen, to form a multilayered vasculature tissue model. Figure 7 (e, f) shows the results of the Live/Dead assay of the cells at Days 3 and 5. We successfully cultured cells in the collagen hydrogel tubes while maintaining high cell viability. The presented approaches would be therefore useful for perfusion cell culture systems that can realize proper cell-matrix interactions.
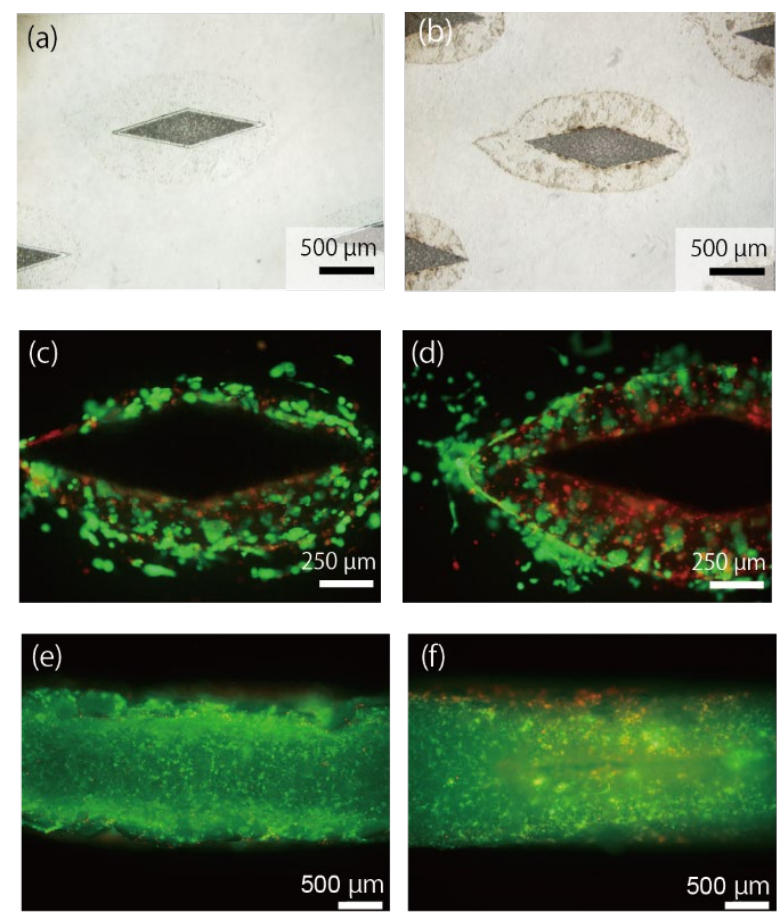

Figure 7. ( $a, b)$ Bright field micrographs showing the HepG2 cell-encapsulating collagen hydrogels at (a) Day 0 and (b) Day 7; (c, d) Live/Dead cell assay for the Hep G2 cells at (c) Day 3 and (d) Day 7; (e, f) Live/Dead cell assay for the cells in the collagen tubes at (e) Day 3 and (f) Day 5

\section{Conclusions}

We have proposed a new approach to precisely fabricating collagen microstructures in microfluidic devices, without necessitating complicated operations or devices. We successfully applied the formed collagen microstructures to the perfusion culture of mammalian cells, which can mimic the circulation environment in our body. The presented strategy would be useful as a variety of perfusion cell culture platforms, including organs-on-chip devices for drug development and basic biological studies.

\section{Acknowledgements}

This study was supported in part by Grants-in-aids for Scientific Research (18K18969 and 17H03463) from the Ministry of Education, Culture, Science, Sports, and Technology of Japan.

\section{References}

Furusawa, K., S. Sato, J. Masumoto, Y. Hanazaki, Y. Maki, T. Dobashi, T. Yamamoto, A. Fukui, and N. Sasaki; "Studies on the Formation Mechanism and the Structure of the Anisotropic Collagen Gel Prepared by DialysisInduced Anisotropic Gelation," Biomacromolecules, 13, 29-39 (2012)

Iwadate, H., M. Yamada, N. Naoki, R. Hashimoto, Y. Yajima, R. Utoh, and M. Seki; "PDMS Microstencil Plate-Supported Fabrication of Ultra-thin, Condensed ECM Membranes for Separated Cell Coculture on both 
Surfaces," Sens. Actuators B: Chem., 287, 486-495 (2019)

Salerno, S., E. Curcio, A. Bader, L. Giorno, E. Drioli, and L. D. Bartolo; "Gas Permeable Membrane Bioreactor for the Co-culture Of Human Skin Derived Mesenchymal Stem Cells with Hepatocytes and Endothelial Cells," J. Membr. Sci., 563, 694-707 (2018)

Walter, F. R., S. Valkai, A. Kincses, A. Petnehazi, T. Czeller, S. Veszelka, P. Ormos, M. A. Deli, and A. Der; "A Versatile lab-on-a-chip Tool for Modeling Biological Barriers," Sens. Actuators B: Chem., 222, 1209-1219 (2016) 